\title{
High Resolution Multibeam Survey and Mobile Laser Scanning - Comprehensive Information for Coastal Infrastructure Management and Planning
}

Jani PÖTRÖNEN, Finland

Hydrographic Survey Engineer, M.Sc.(Surveying)

Topic A: innovations in acquisition techniques

\section{INTRODUCTION}

The use of professional divers is the most common method used in the inspection of underwater structures nowadays. However, comprehensive dive inspections are very time consuming and the results may consist of not much more than drawings, fuzzy photographs, and written text. In many locations poor visibility and certain environmental conditions can substantially lower the scope of such inspection or even make it impossible.

This paper presents how high resolution multibeam (MBES) survey, mobile laser scanning and scanning sonar technology can be used in an effective way to gather comprehensive information of your coastal infrastructure above and below the water surface. The benefits compared to other commonly used techniques to gather the information will also be outlined.

\section{MULTIBEAM SURVEY}

Multibeam survey is commonly used for hydrographic charting. Technical development with these sensors has been fast. Nowadays it is possible to collect a huge number of high resolution survey points of not only from the natural seabed but also from other kind of underwater objects. This way much smaller targets can be located and identified underwater.

Multibeam survey is the most economic and effective way to get an extensive underwater view of coastal structures. By tilting the multibeam sonar head sideways, underwater 3D data can be collected from the bottom up to the water surface level. This introduces new possibilities to inspect harbour structures and other underwater civil engineering targets more comprehensively.

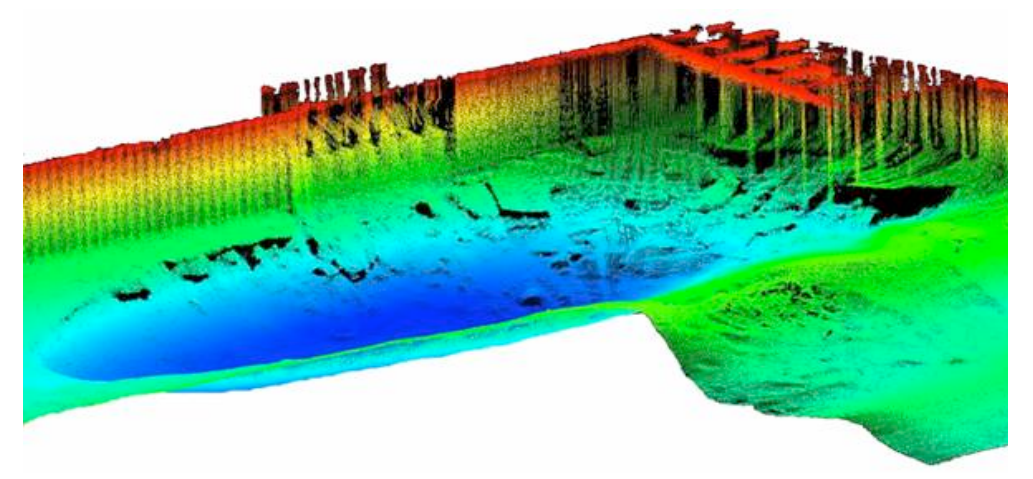

Figure 1: Multibeam survey dataset by tilted sonar head from Port of Helsinki.

High resolution multibeam data shows valuable information about possible slope failures, mass movements due to propeller race and condition of erosion protection in front of quay walls. Also missing objects like containers and quay wall fenders can be located from the data with high accuracy beside with possible hazards for save navigation in the harbour. The survey results as 3D point clouds are compatible with all modern software for designing, planning and engineering purposes. 
Laser scanning is a state of the art method to collect a 3D point cloud of a certain area of interest in extremely high detail level. When mobile laser scanning is carried out simultaneously with the multibeam survey from a modern hydrographic survey vessel, this makes it a highly cost effective way of working.

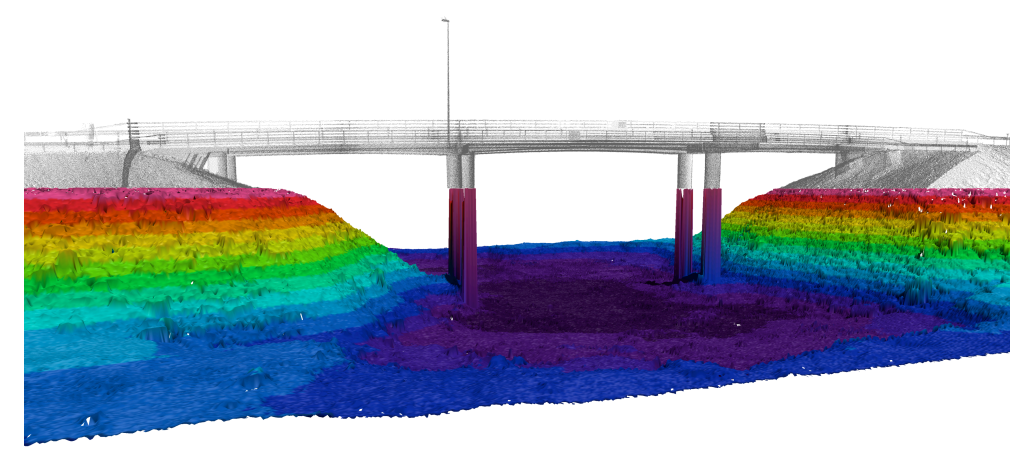

Figure 2: Combined multibeam and laser scanning dataset from Särkisalo bridge.

Combining diver's observations and photographs in a paper report is not compatible to what you can achieve by combining mobile laser scanning of coastal infrastructure with multibeam point cloud of underwater structures. All the objects located in the dataset have exact coordinates and are easy to locate for further inspection through comprehensive view of the area of interest above and below the water surface. This kind of dataset also allows you to document your complete infrastructure for the future development needs.

\section{SCANNING SONAR}

Scanning sonar is the most accurate method to make supplementary inspections for underwater structures in strong current or turbid waters where diving is not possible. Scanning sonar operates at high frequency typically from $600 \mathrm{kHz}$ which gives a good resolution.

Scanning sonar data can be accurate 2D images or 3D point clouds tied to a coordinate system. Any kind of damage or structural deviations as well as their location and extent can be defined in the inspection.

Depending on the circumstances at the site, the inspections can be carried out from a barge, crane or even from frozen sections of water. Compared to multibeam data the resolution of the data is better when sonar mount is static and this way recognized potential targets can be inspected more carefully to do the repair planning.

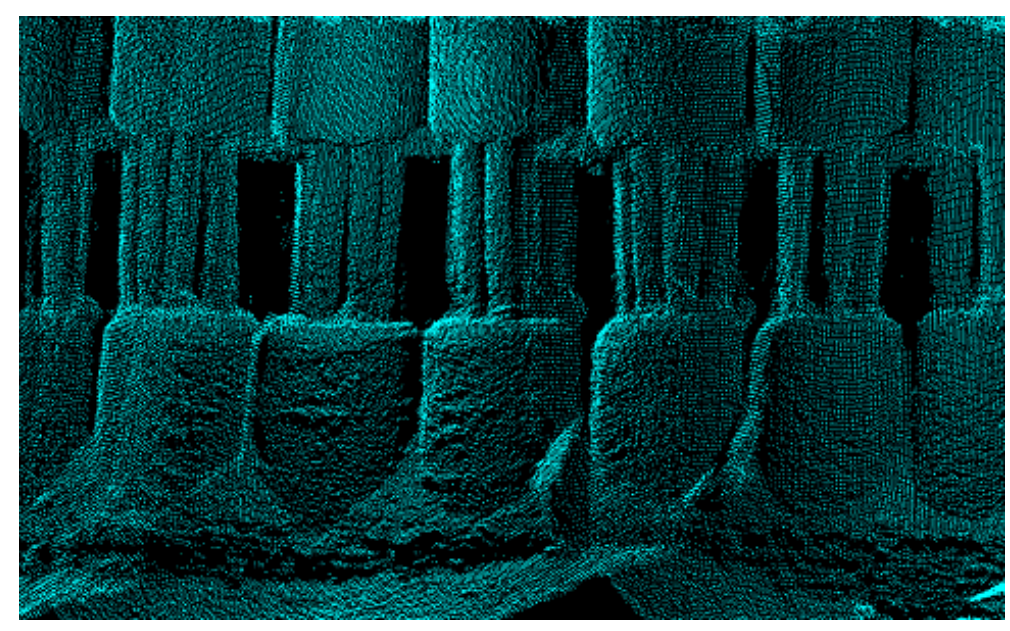

Figure 3: Scanning sonar dataset of the piles (VRT Finland Ltd). 


\section{CONCLUSION}

Multibeam survey and mobile laser scanning provide us a tool to obtain a comprehensive view of a location on a larger scale, and to recognize potential targets for further inspection. These specific targets can then be inspected more closely for example by professional divers. Alternatively, scanning sonar technology offers the most accurate method to inspect underwater structures locally without the need to worry about visibility or strong current.

For a professional inspection of coastal infrastructure the most effective solution is a combination of simultaneous multibeam survey and mobile laser scanning combined with focused scanning sonar inspection. All information in 3D digital format is then usable in modern software for further maintenance planning and engineering purposes. This approach will help to safeguard maritime investments and improve maintenance planning in your harbours and other marine infrastructure.

\section{CONTACT DETAILS \\ Jani PÖTRÖNEN \\ Meritaito Ltd \\ Porkkalankatu 5 \\ FI-00180 Helsinki \\ FINLAND}

Tel: +358207030300

GSM: +358 405292748

Fax: +358 207030399

Email: jani.potronen@meritaito.fi

Web site: www.meritaito.fi/www/en 